\title{
The heat shock protein-90 co-chaperone, Cyclophilin 40, promotes ALK-positive, anaplastic large cell lymphoma viability and its expression is regulated by the NPM-ALK oncoprotein
}

\author{
Joel D Pearson ${ }^{1}$, Zubair Mohammed ${ }^{1}$, Julinor T C Bacani ${ }^{2}$, Raymond Lai ${ }^{2}$ and Robert J Ingham ${ }^{\text {1* }}$
}

\begin{abstract}
Background: Anaplastic lymphoma kinase-positive, anaplastic large cell lymphoma (ALK+ ALCL) is a T cell lymphoma defined by the presence of chromosomal translocations involving the ALK tyrosine kinase gene. These translocations generate fusion proteins (e.g. NPM-ALK) with constitutive tyrosine kinase activity, which activate numerous signalling pathways important for ALK+ ALCL pathogenesis. The molecular chaperone heat shock protein-90 (Hsp90) plays a critical role in allowing NPM-ALK and other signalling proteins to function in this lymphoma. Co-chaperone proteins are important for helping Hsp90 fold proteins and for directing Hsp90 to specific clients; however the importance of co-chaperone proteins in ALK+ ALCL has not been investigated. Our preliminary findings suggested that expression of the immunophilin co-chaperone, Cyclophilin 40 (Cyp40), is up-regulated in ALK+ ALCL by JunB, a transcription factor activated by NPM-ALK signalling. In this study we examined the regulation of the immunophilin family of co-chaperones by NPM-ALK and JunB, and investigated whether the immunophilin co-chaperones promote the viability of ALK+ ALCL cell lines.

Methods: NPM-ALK and JunB were knocked-down in ALK+ ALCL cell lines with siRNA, and the effect on the expression of the three immunophilin co-chaperones: Cyp40, FK506-binding protein (FKBP) 51, and FKBP52 examined. Furthermore, the effect of knock-down of the immunophilin co-chaperones, either individually or in combination, on the viability of ALK+ ALCL cell lines and NPM-ALK levels and activity was also examined.
\end{abstract}

Results: We found that NPM-ALK promoted the transcription of Cyp40 and FKBP52, but only Cyp40 transcription was promoted by JunB. We also observed reduced viability of ALK+ ALCL cell lines treated with Cyp40 siRNA, but not with siRNAs directed against FKBP52 or FKBP51. Finally, we demonstrate that the decrease in the viability of ALK+ ALCL cell lines treated with Cyp40 siRNA does not appear to be due to a decrease in NPM-ALK levels or the ability of this oncoprotein to signal.

Conclusions: This is the first study demonstrating that the expression of immunophilin family co-chaperones is promoted by an oncogenic tyrosine kinase. Moreover, this is the first report establishing an important role for Cyp40 in lymphoma.

\footnotetext{
* Correspondence: ringham@ualberta.ca

${ }^{1}$ Department of Medical Microbiology and Immunology, University of

Alberta, Edmonton T6G 2E1, Canada

Full list of author information is available at the end of the article
} 


\section{Background}

Anaplastic lymphoma kinase-positive, anaplastic large cell lymphoma (ALK+ ALCL) is an aggressive non-Hodgkin lymphoma of $\mathrm{T} /$ null cell immunophenotype [1-3]. This lymphoma primarily presents in children, adolescents, and young adults where it accounts for $10-20 \%$ of childhood non-Hodgkin lymphomas [1]. ALK+ ALCL is characterized by the presence of chromosomal translocations involving the $A L K$ gene, which encodes for a receptor tyrosine kinase belonging to the insulin receptor super-family. These translocations result in the expression of ALK fusion proteins that are critical for the pathogenesis of ALK+ ALCL [2,3]. Moreover, ALK fusion proteins have been implicated in the pathogenesis of a subset of non-small cell lung carcinomas (ALK+ NSCLC) [4-7] and inflammatory myofibroblastic tumours (ALK+ IMT) [8-10]. In ALK+ ALCL several different $A L K$ translocations have been described [2,3]; however, the most common $(\sim 80 \%)$ is the $\mathrm{t}(2 ; 5)(\mathrm{p} 23 ; \mathrm{q} 35)$ translocation involving the nucleophosmin (NPM) gene which generates the NPM-ALK oncogene [1-3].

NPM-ALK consists of the N-terminal region of NPM and the C-terminal kinase and intracellular domains of ALK [11,12]. The NPM portion of this fusion protein possesses a dimerization domain required for the tyrosine kinase activity and transforming ability of NPMALK $[13,14]$. The activity of the NPM-ALK oncoprotein is also critically dependent on the molecular chaperone, heat shock protein-90 (Hsp90) [15-18]. Hsp90 is a ubiquitously expressed protein that assists in the proper folding and activity of numerous cellular proteins $[19,20]$. Hsp90 promotes the stability of NPM-ALK [15-18], as treatment of cell lines with the Hsp90 inhibitor, 17-Allylamino-Demethoxygeldanamycin (17-AAG), resulted in the proteasomal degradation of NPM-ALK [17]. The treatment of ALK+ ALCL cell lines with 17AAG resulted in cell cycle arrest and the induction of apoptosis $[15,18]$; however, these effects are likely due to more than just decreased NPM-ALK levels. Hsp90 inhibition also decreased levels of the pro-survival serine/ threonine kinase Akt, the cell cycle-associated proteins cyclin D1, cyclin-dependent kinase 4 (cdk4), and cdk6, as well as several other proteins in ALK+ ALCL $[15,18,21]$. The treatment of ALK+ ALCL cell lines with 17-AAG resulted in decreased phosphorylation of the serine/threonine kinase Erk without affecting Erk levels [15]. Moreover, the treatment of ALK+ NSCLC with Hsp90 inhibitors resulted in Erk dephosphorylation as well as the degradation of Akt and the EML4-ALK oncoprotein in these tumours [22-24].

Hsp90 inhibitors are also effective at inhibiting EML4ALK-driven tumourigenesis in vivo in the mouse [22,23], and the treatment of three ALK+ NSCLC patients with the Hsp90 inhibitor, IPI-504, resulted in a partial response in two of the patients and stable disease in the other [25]. Importantly, Hsp90 inhibitors are effective against tumour cells expressing ALK fusion proteins that possess mutations that render them resistant to the ALK inhibitor, Crizotinib [24,26]. Thus, Hsp90 inhibitors may be useful in treating patients that develop resistance to ALK inhibitors.

One aspect of Hsp90 biology that is largely unstudied in ALK-expressing tumours is the role of Hsp90 cochaperones. Many functions of Hsp90 are dependent on its association with co-chaperone proteins $[19,20]$. Cochaperones mediate various aspects of Hsp90 function, including the association of Hsp90 with client proteins and the regulation of Hsp90 ATPase activity $[19,20]$. Cyclophilin 40 (Cyp40), FK506-binding protein (FKBP) 51, and FKBP52 are members of the immunophilin family of Hsp90 co-chaperones. This family is best characterized for its association with Hsp90-steroid hormone receptor complexes containing client proteins such as the glucocorticoid, estrogen, progesterone, and androgen receptors [27-30]. The individual immunophilin family members show some preference for specific hormone receptors, and they can both antagonize and promote the transcription mediated by these receptors. For example, FKBP51 inhibits the transcriptional activity of the glucocorticoid receptor [31-33], while FKBP52 is important for promoting the transcriptional activity of this receptor [32-35]. In addition to steroid hormone receptors, immunophilin co-chaperones have been found to complex with the Lck [36] and Fes [37] tyrosine kinases. As well, the expression and activity of ectopically expressed $\mathrm{v}$-Src oncoprotein in Saccharomyces cerevisiae is dependent on the Cyp40 homolog, Cpr7 [38]. Immunophilin co-chaperones are important in cancer, as Cyp40 and FKBP51 have been shown to promote the proliferation of androgen-dependent and androgenindependent prostate cancer cell lines [39].

We identified Cyp40 in a mass spectrometry screen designed to identify proteins regulated by the JunB transcription factor in ALK+ ALCL (R.J.I and J.D.P; unpublished observation). JunB is an AP-1 family transcription factor that is highly expressed in ALK+ ALCL [40-42], and has been shown to promote the proliferation of the Karpas 299 ALK+ ALCL cell line [43]. This transcription factor also promotes the expression of CD30 [44,45] and the cytotoxic protein, Granzyme B [46], in ALK+ ALCL, which are phenotypic characteristics of this lymphoma $[1,47]$. Since co-chaperone proteins are important for Hsp90 function, and Hsp90 activity is critical in ALK+ ALCL, we were intrigued by our observation that JunB might promote the expression of Cyp40 in ALK+ ALCL. In this study, we examined whether the expression of the immunophilin co-chaperones was regulated by oncogenic signalling in ALK+ ALCL. We also investigated 
whether the immunophilin co-chaperone proteins were important for the viability of ALK+ ALCL cell lines. We found that NPM-ALK induced the transcription of two immunophilin family co-chaperones, Cyp40 and FKBP52, but that only Cyp40 transcription was promoted by JunB. In addition, knocking-down the expression of Cyp40, but not FKBP51 or FKBP52, reduced the viability of ALK+ ALCL cell lines. However, knock-down of the immunophilin proteins did not appear to regulate NPM-ALK stability or activation. In conclusion, we demonstrate that some members of the immunophilin family of Hsp90 co-chaperone proteins are targets of NPM-ALK signalling, and that Cyp40 plays an important role(s) in ALK+ ALCL that is not shared by other immunophilin family co-chaperones.

\section{Methods}

\section{Reagents and CDNA constructs}

The monoclonal antibodies (mAbs) against JunB (C-11 and 204C4a), FKBP51, FKPB52, STAT3, phospho-STAT3 (Tyr 705), Myc, and $\beta$-actin were from Santa Cruz Biotechnology (Santa Cruz, CA). The Cyp40 polyclonal antibody was also from Santa Cruz Biotechnology. The anti-JunB (C-11) mAb was used for western blotting, while the anti-JunB (204C4a) mAb was used in EMSA experiments. The anti-tubulin $\mathrm{mAb}$ was from Calbiochem (San Diego, CA), the anti-ALK mAb from Dako (Burlington, ON, Canada), and the anti-phosphotyrosine mAb (4G10) was from Millipore (Billerica, MA). Antiphospho-ALK (Tyr 338, 342, and 343 of NPM-ALK) and anti-Akt antibodies were purchased from Cell Signalling Technology (Danvers. MA). Short interfering RNA (siRNA) oligonucleotides were purchased from Dharmacon RNAi Technologies (Lafayette, CO). The NPM-ALK inhibitor, Crizotinib, was generously provided by Pfizer $[7,48,49]$. To generate the human Cyp40 promoterdriven luciferase reporter construct, we PCR amplified the Cyp 40 proximal promoter $(-691$ to +62 relative to the transcriptional start site) from the Karpas 299 cell line and cloned it into the pGL2 basic luciferase vector (Promega; Madison, WI). The AP-1 consensus sequence in the Cyp 40 promoter was mutated from TGATTCA to TAACTAA to generate the AP- 1 mutant construct. The Myc-tagged JunB construct was generated by adding a double myc tag to the $5^{\prime}$ end of the human JunB cDNA. This was then cloned into the pcDNA 3.1A eukaryotic expression vector (Invitrogen; Burlington, ON, Canada).

\section{Cell lines and electroporations}

The Karpas 299 and SUP-M2 ALK+ ALCL cell lines were cultured in RPMI 1640 supplemented with 10\% heat-inactivated FBS, $2 \mathrm{mM}$ L-glutamine, $1 \mathrm{mM}$ sodium pyruvate, and $50 \mu \mathrm{M}$ 2-mercaptoethanol. For transfections involving siRNAs, $4 \times 10^{6}$ cells were transfected by electroporation with $100 \mathrm{nM}$ pooled siRNA as previously described [50]. Cells were then incubated for $48 \mathrm{~h}$ at $37^{\circ}$ $\mathrm{C}$ prior to analysis. For luciferase reporter assays, $1 \times 10^{7}$ cells were transfected with $10 \mu \mathrm{g}$ of the indicated pGL2 luciferase construct and $1 \mu \mathrm{g}$ of a constitutively expressed Renilla luciferase construct (to control for transfection efficiency). In luciferase experiments involving siRNAs, cells were also transfected with $100 \mathrm{nM}$ pooled control (non-targeting) or JunB siRNA. For luciferase assays performed on Karpas 299 cells over-expressing JunB, cells were transfected with the luciferase constructs as described above and $5 \mu \mathrm{g}$ of Myc-tagged JunB or empty vector. Cells were then incubated for $24 \mathrm{~h}$ at $37^{\circ} \mathrm{C}$ prior to analysis of luciferase activity (see below).

\section{Cell lysis, immunoprecipitations, and western blotting}

Cells were lysed in Nonidet P-40 lysis buffer [50] containing protease inhibitor cocktail (Sigma-Aldrich; Mississauga, ON, Canada), $1 \mathrm{mM}$ phenylmethylsulfonylfluoride, and $1 \mathrm{mM}$ sodium orthovanadate. Lysates were cleared of detergent-insoluble material by centrifugation at $\sim 20,000 \mathrm{~g}$ for $10 \mathrm{~min}$. The protein concentration of cleared lysates was determined using the BCA Protein Assay kit (Thermo Scientific; Waltham, MA). Anti-ALK immunoprecipitations were performed by incubating cleared lysates with $0.5 \mu \mathrm{g}$ of the anti-ALK antibody and Protein A-Sepharose beads (Sigma-Aldrich) for 1-2 $\mathrm{h}$ at $4^{\circ} \mathrm{C}$ on a nutator. Beads were subsequently washed with lysis buffer and bound proteins eluted by boiling in SDSPAGE sample buffer. Cell lysates or immunoprecipitates were resolved on SDS-PAGE gels and transferred to nitrocellulose membranes. Western blots were visualized using SuperSignal West Pico Chemiluminescent Substrate (Thermo Scientific) and band intensities quantified using a LI-COR Odyssey Infrared Imager (LI-COR Biosciences; Lincoln, NE). Expression of the quantified proteins were normalized to tubulin levels and expressed relative to control (non-targeting) siRNA-treated cells. The number of independent replicates for each experiment are indicated in the figure legends. To reprobe blots, membranes were stripped in 0.1\% TBST, pH 2 prior to incubation with the new primary antibody.

\section{Quantitative RT-PCR (qRT-PCR)}

After collection using the RNeasy mini kit (Qiagen; Mississauga, ON), total RNA was digested with DNase I to remove potential DNA contamination, and then reverse transcribed to cDNA using the Superscript II Reverse Transcriptase System (Invitrogen; Burlington, ON, Canada). qRT-PCR was performed using PerfeCTa SYBR Green FastMix (Quanta Biosciences; Gaithersburg, MD) on an Eppendorf Mastercycler realplex ${ }^{4}$ thermal cycler. Cyp40 and FKBP52 mRNA levels were then determined using the $\triangle \Delta$-CT method [51] with $\beta$-actin as the 
housekeeping gene. The following primers were used: Cyp40 forward - TCGAGTCTTCTTTGACGTGGA, reverse - CAGTCGTGTGTCCAATGCCTT; FKBP52 forward - TGCTGAAGGTCATCAAGAGAGAG, reverse - ATGGTGGCTATGGCAATGTC; actin forward AGAAAATCTGGCACCACACC, reverse - TAGCACAGCCTGGATAGCAA. Results are displayed relative to control siRNA-transfected cells and represent the mean and standard deviation of three independent experiments.

\section{Luciferase assays}

Luciferase assays were performed on a BMG Labtech Plate Reader using the Dual-Glo Luciferase Assay System (Promega) and the protocol provided by the manufacturer. Cyp40 promoter-driven firefly luciferase and constitutive Renilla luciferase activity were determined in triplicate for each sample. The level of firefly activity was normalized to Renilla activity and triplicate measurements were averaged. Three independent replicates were performed for each experiment.

\section{Electrophoretic mobility shift assay (EMSA)}

Nuclear extracts were collected from Karpas 299 cells using the ProteoJET cytoplasmic and nuclear protein extraction kit (Fermentas; Burlington, ON, CA). EMSAs were performed with the LightShift chemiluminescent EMSA kit (Thermo Scientific) using a biotinylated probe corresponding to a 20 nucleotide sequence surrounding the AP-1 site of the Cyp40 promoter (TTGTACTGATTCATGTCTTT). The unlabeled AP-1 mutant competitor contained the same mutation as described for the luciferase reporter construct (see above). Binding reactions were performed with $7.5 \mu \mathrm{g}$ of nuclear protein extract, $100 \mathrm{fmol}$ of the Cyp40 promoter probe, and a 50-fold molar excess of an unlabeled Cyp 40 promoter as a competitor. For super-shift experiments, $1 \mu \mathrm{g}$ of the indicated antibody was pre-incubated with the reaction mixture for 15 min on ice prior to addition of the biotinylated probe.

\section{MTS viability assays}

After transfection with the indicated siRNAs, cells were resuspended to $4 \times 10^{4}$ cells $/ \mathrm{ml}$ and incubated at $37^{\circ} \mathrm{C}$ for $48 \mathrm{~h}$. The number of viable cells in each sample was determined in triplicate using the CellTiter 96 AQueous Non-Radioactive Cell Proliferation Assay (MTS assay) (Promega). Triplicate measurements were then averaged and the percentage of viable cells determined relative to cells transfected with control siRNA. Each experiment was performed in quadruplicate.

\section{Statistical analysis}

Statistical analysis was performed using paired, onetailed $t$-test in all cases, except the comparison of viability with Cyp40 siRNA to combined siRNA in which an unpaired, one-tailed $t$-test was performed.

\section{Results}

JunB promotes Cyp40, but not FKBP51 or FKBP52, expression in ALK+ ALCL cell lines

To confirm our mass spectrometry findings showing that JunB promotes the expression of Cyp40 in ALK+ ALCL, we performed western blotting experiments. Despite incomplete JunB knock-down, we observed a decrease in Cyp40 protein expression after knock-down of JunB with siRNA in both the Karpas 299 and SUP-M2 ALK+ ALCL cell lines (Figure 1A). Since Cyp40 belongs to the immunophilin family of Hsp90 co-chaperone proteins, which includes FKBP51 and FKBP52, we also examined whether JunB promotes the expression of these proteins. However, we found that JunB knockdown did not influence FKBP51 or FKBP52 protein expression in ALK+ ALCL cell lines (Figure 1B and C).

We next examined Cyp40 mRNA levels after treatment of cells with JunB siRNA, and found that knockdown of JunB resulted in decreased levels of Cyp40 mRNA in both Karpas 299 and SUP-M2 cells (Figure 2A). We also generated a luciferase reporter construct where expression of firefly luciferase is under control of the human Cyp40 promoter. When transfected into Karpas 299 cells this construct exhibited strong luciferase activity, which was reduced when cells were co-transfected with JunB siRNA (Figure 2B). In addition, over-expression of Myc-tagged JunB was found to promote transcription from this luciferase promoter construct, further demonstrating that JunB promotes transcription of Cyp 40 (Figure 2C). The Cyp 40 promoter contains a consensus sequence for AP-1 family transcription factors [52] that could be recognized by JunB. Mutation of this site resulted in reduced luciferase activity (Figure 2D), demonstrating this site is important for Cyp40 transcription. To examine whether JunB can bind this AP-1 site we performed EMSA experiments (Figure 2E). We found that a protein(s) expressed by Karpas 299 cells bound to a biotinylated probe corresponding to the AP-1 site in the Cyp40 promoter. We further found that JunB was a major component of the probe/protein complex(es) bound to this AP-1 site, as inclusion of an anti-JunB antibody in the binding reaction resulted in an almost complete super-shift of the probe/protein complex. Taken together, our results argue that JunB functions as a direct transcriptional activator of Cyp 40 in ALK+ ALCL.

\section{NPM-ALK promotes Cyp40 and FKBP52, but not FKBP51, expression}

The NPM-ALK oncoprotein drives much of the signalling underlying the pathogenesis of ALK+ ALCL [2,3], 

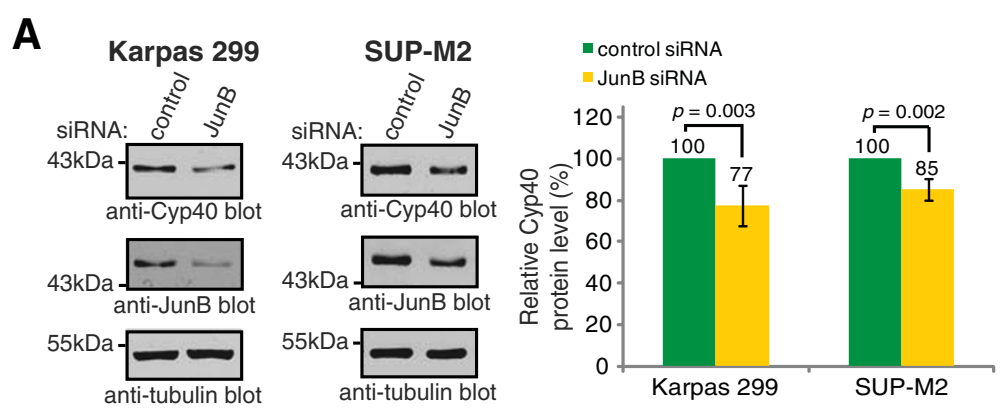

B
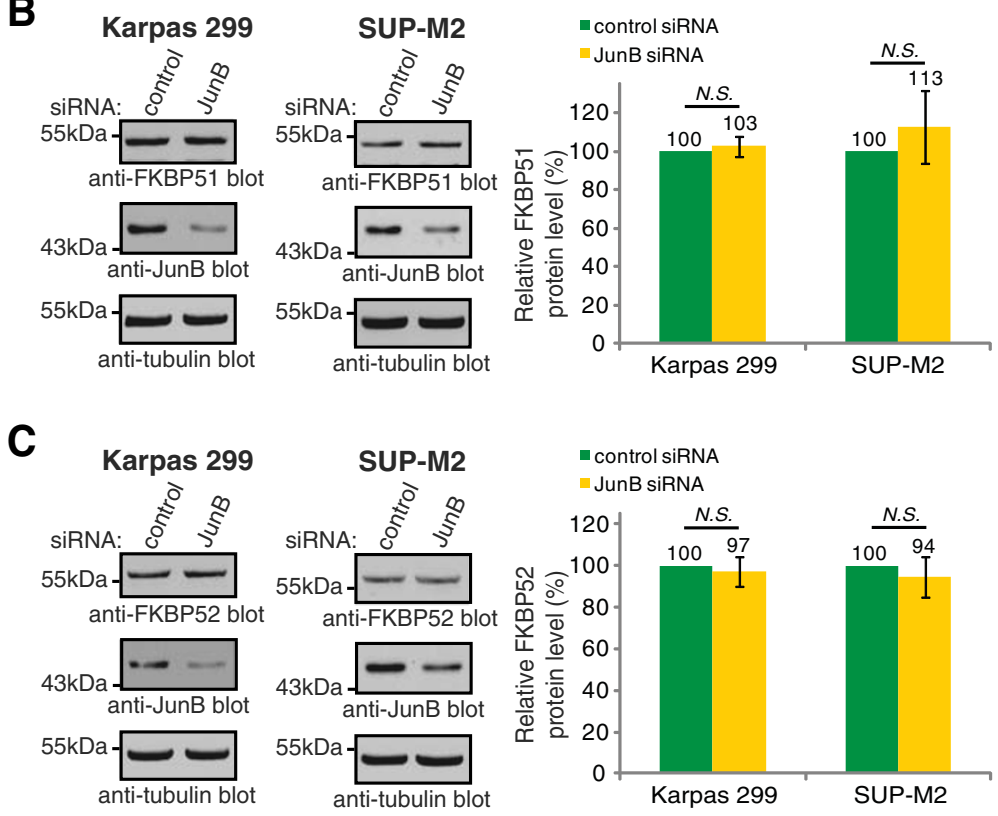

Figure 1 JunB promotes Cyp40, but not FKBP51 or FKBP52, protein expression in ALK+ ALCL. Western blot analysis (left) and quantification (right) of Cyp40 (A), FKBP51 (B), and FKBP52 (C) levels in lysates collected from Karpas 299 and SUP-M2 cells treated with pooled control (non-targeting) or JunB siRNA. Quantification represents the mean and standard deviation of five (Cyp40) or four (FKBP51 and FKBP52) independent experiments. $p$ values were obtained using paired, one-tailed t-tests. N.S. indicates a $p$ value $>0.05$.

including the elevated expression of JunB $[43,44,46]$. Therefore, we next examined whether NPM-ALK promotes expression of the immunophilin co-chaperones in ALK+ ALCL. We found that knock-down of NPM-ALK in Karpas 299 and SUP-M2 cells resulted in significantly reduced Cyp40 protein levels (Figure 3A). NPM-ALK knock-down also resulted in a substantial reduction in JunB levels, that was comparable to the reduction in JunB observed after JunB siRNA treatment (compare Figure 3A and Figure 1). Knock-down of NPM-ALK also resulted in decreased FKBP52 expression, but had no effect on the expression of FKBP51 (Figures 3B and C, respectively). Using quantitative RT-PCR, we found that knock-down of NPM-ALK reduced Cyp40 (Figure 3D) and FKBP52 (Figure 3E) mRNA expression in ALK+ ALCL cell lines. These findings show that both Cyp 40 and FKBP52 are transcriptional targets of NPM-ALK signalling in ALK+ ALCL.
To further examine the regulation of the immunophilin co-chaperones by NPM-ALK, we treated ALK+ ALCL cell lines with the ALK inhibitor, Crizotinib, which has been shown to be useful in treating patients with ALK+ ALCL [53] and EML4-ALK+ NSCLC [7,54,55]. Treatment of Karpas 299 and SUP-M2 cells with Crizotinib resulted in a dose- (Figure $4 \mathrm{~A}$ ) and timedependent (Figure 4B) decrease in NPM-ALK phosphorylation on tyrosines 338, 342, and 343. These phosphorylation sites are located within the activation loop of the kinase domain, and their phosphorylation correlates with NPM-ALK activation [56,57]. Furthermore, we observed a dose- and time-dependent decrease in Cyp40 and FKBP52 protein expression in both Karpas 299 and SUP-M2 cells after Crizotinib treatment (Figure $4 \mathrm{C}$ and D). In contrast, Crizotinib treatment did not decrease FKBP51 expression in either cell line; however it did result in a modest, but reproducible, 
A

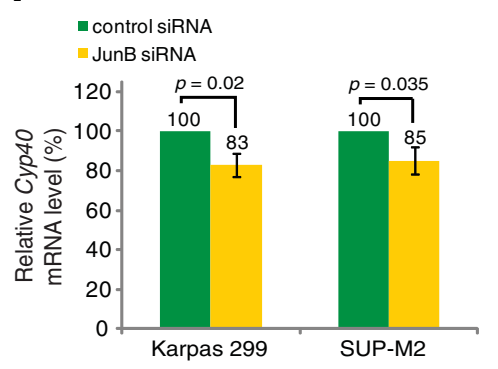

C

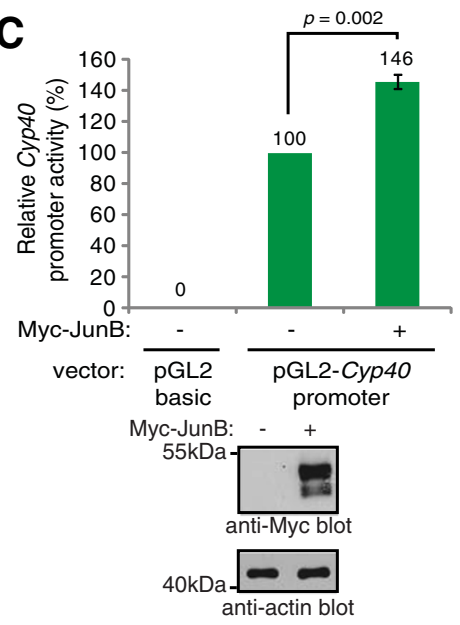

D

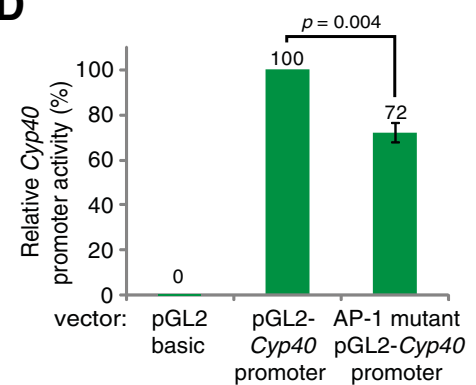

B

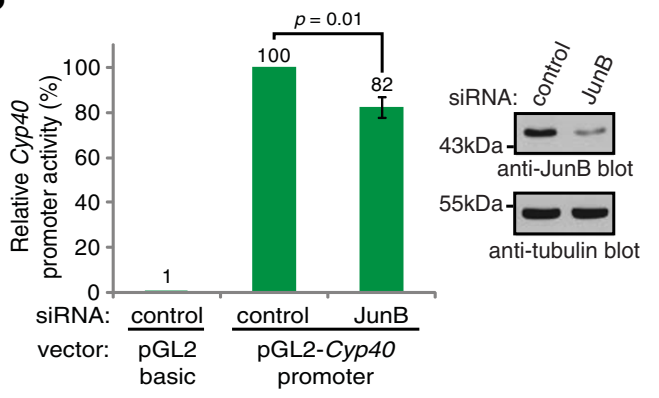

E

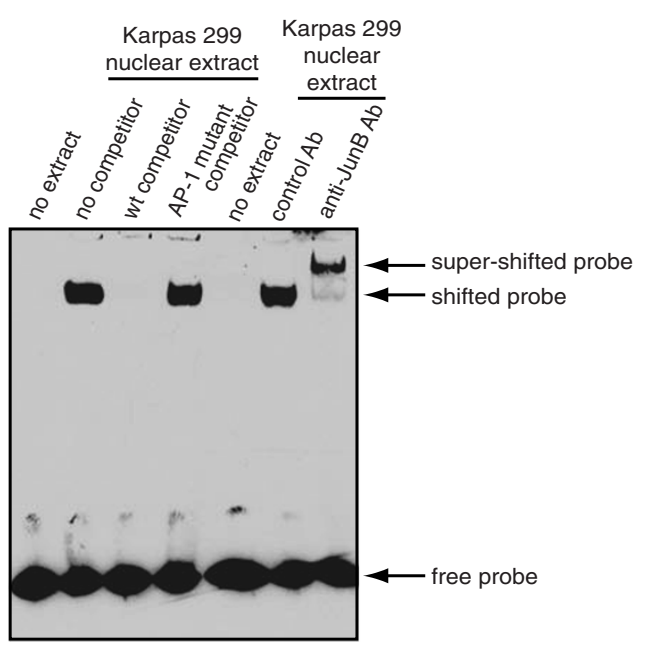

Figure 2 JunB promotes Cyp40 transcription in ALK+ ALCL. (A) qRT-PCR analysis of Cyp40 mRNA levels in Karpas 299 and SUP-M2 cells transfected with pooled control or JunB siRNA. (B) Luciferase activity in Karpas 299 cells transfected with a human Cyp40 promoter-driven (pGL2-Cyp40 promoter) or promoter-less (pGL2 basic) luciferase vector, and pooled control or JunB siRNA (left). Results are presented relative to the activity present in cells co-transfected with pGL2-Cyp40 promoter and control siRNA. A western blot demonstrating the level of JunB silencing is shown (right). (C) Luciferase activity in Karpas 299 cells transfected with the luciferase constructs described in (A) along with empty vector (-) or Myc-tagged JunB (+) (top). A western blot illustrating the expression of the Myc-tagged JunB is also included (bottom). (D) Luciferase activity in Karpas 299 cells transfected with the pGL2 basic, pGL2-Cyp40 promoter, or AP-1 mutant PGL2-Cyp40 promoter luciferase construct. Luciferase activity is expressed relative to the activity present in the PGL2-Cyp40 promoter transfected cells. (E) EMSAs were performed using a biotinylated Cyp40 AP-1 probe and no competitor, an unlabeled Cyp40 AP-1 competitor (wt competitor), an unlabeled Cyp40 competitor with a mutation in the AP-1 site (AP-1 mutant competitor) or the anti-JunB or isotype control (control) antibody (Ab). In all experiments, error bars represent the standard deviation of three independent experiments. $p$ values were determined using paired, one-tailed $t$-tests.

increase in FKBP51 expression in the Karpas 299 cells at low Crizotinib doses (Figure 4C). Thus, similar to our NPM-ALK knock-down results, treatment of ALK+ ALCL cell lines with an NPM-ALK inhibitor resulted in reduced Cyp40 and FKBP52, but not FKBP51, expression.
Knock-down of Cyp40 reduces the viability of ALK+ ALCL cell lines

Hsp90 is vitally important for the proliferation and survival of ALK+ ALCL cell lines $[15,18]$, and is required for the expression and/or activation of important signalling proteins in this lymphoma [15-18,21]. Therefore, we 

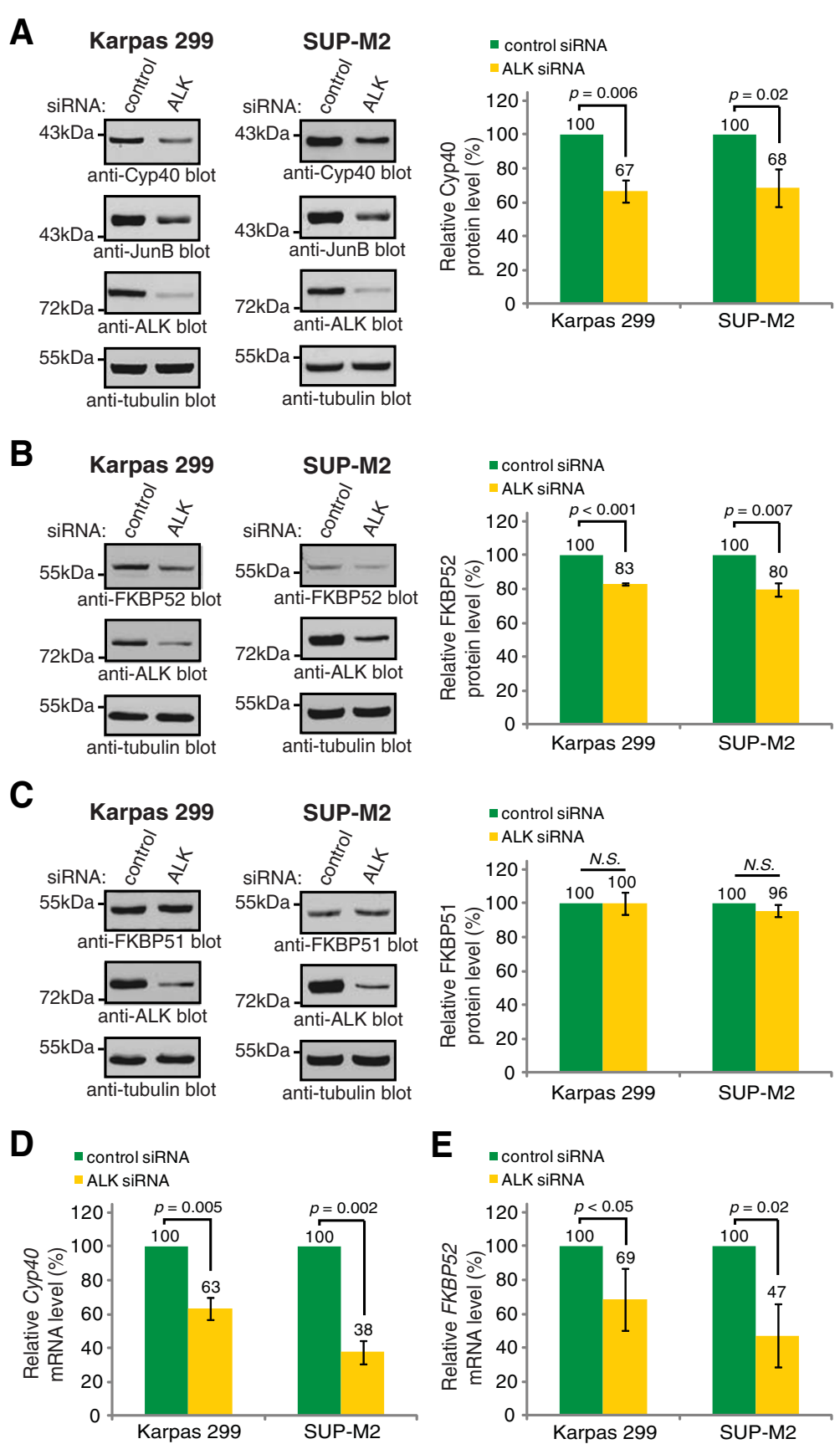

Figure 3 NPM-ALK promotes Cyp40 and FKBP52, but not FKBP51, expression in ALK+ ALCL. Western blot analysis (left) and quantification (right) of Cyp40 (A), FKBP52 (B), and FKBP51 (C) protein levels in Karpas 299 and SUP-M2 cells transfected with pooled control or ALK siRNA. qRTPCR analysis of Cyp40 (D) and FKBP52 (E) mRNA expression in Karpas 299 and SUP-M2 cells transfected with pooled control or ALK siRNA. Error bars represent the standard deviation of the mean of three independent experiments. $p$ values were obtained using paired, one-tailed $t$-tests. N.S. indicates a $p$ value $>0.05$.

examined whether the immunophilin co-chaperones were similarly important in ALK+ ALCL by examining the effect of their knock-down on cellular viability. Treatment of cells with Cyp40 siRNA resulted in a significant reduction in viability in both Karpas 299 and SUP-M2 cells as measured by MTS assay (Figure 5A).
However, we found that reducing the expression of either FKBP51 or FKBP52 did not affect the viability of these cell lines (Figure 5A). The immunophilin cochaperones associate with some of the same Hsp90client protein complexes [37,58,59]; therefore, we examined whether knock-down of FKBP51 and FKBP52 in 
combination with Cyp40 resulted in a greater reduction in viability compared to knock-down of Cyp40 alone. Knock-down of all three immunophilin family members in combination did not significantly reduce viability over
Cyp40 knock-down alone in Karpas 299 and SUP-M2 cells (Figure 5B). This finding argues that the reduced viability observed in these cell lines is predominantly due to decreased Cyp40 expression.

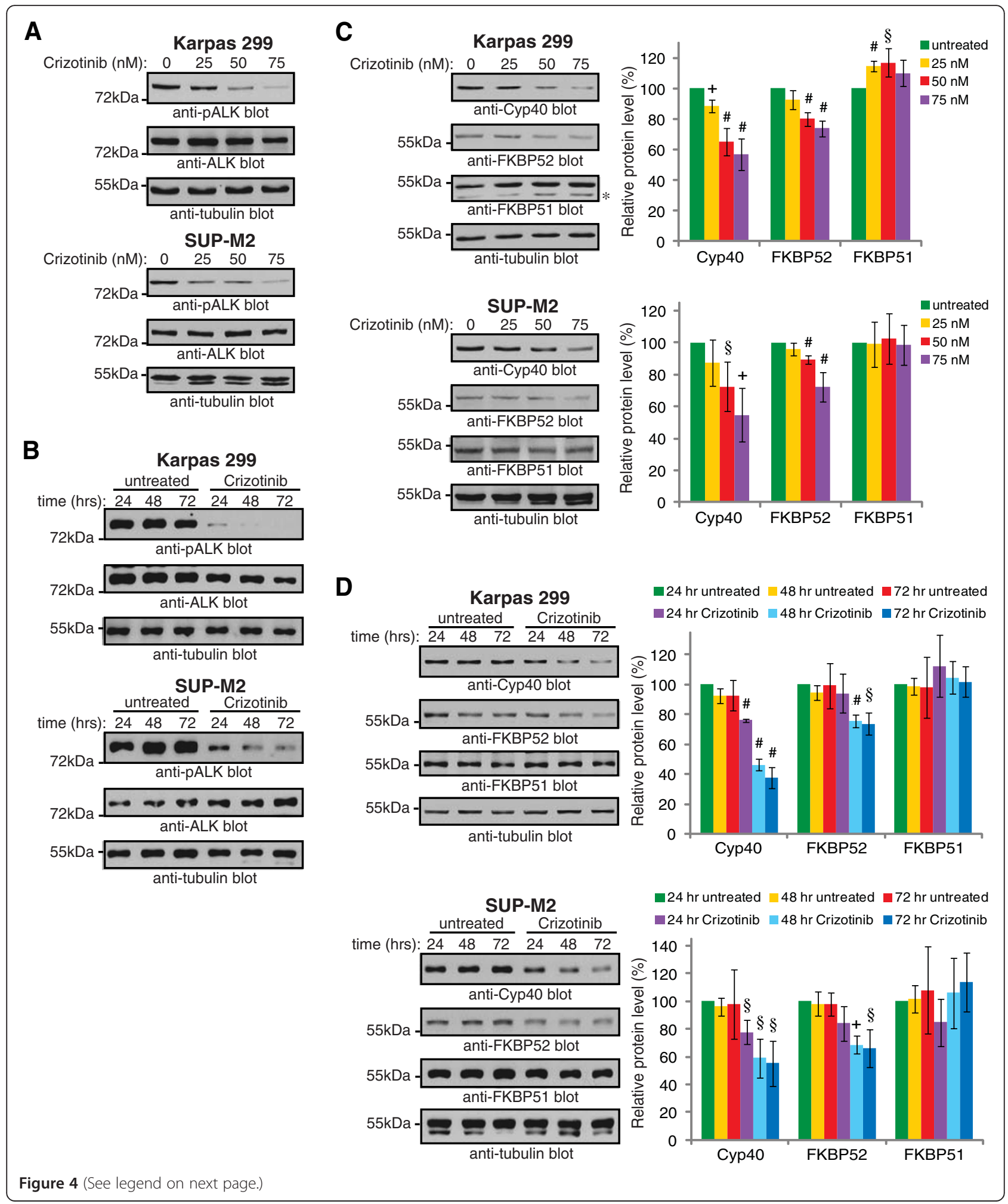


(See figure on previous page.)

Figure 4 Inhibition of NPM-ALK activity results in reduced Cyp40 and FKBP52 protein levels. (A) Western blot analysis examining NPMALK phosphorylation (anti-pALK blot) in Karpas 299 (upper) or SUP-M2 (lower) cells left untreated or treated with 25,50 or 75 nM of the ALK inhibitor, Crizotinib, for 48 h. (B) NPM-ALK phosphorylation in Karpas 299 (upper) or SUP-M2 (lower) cells left untreated or treated with 75 nM Crizotinib for 24, 48 or $72 \mathrm{~h}$. Note: all cells were split into fresh Crizotinib-containing media 24 and $48 \mathrm{~h}$ post-treatment to maintain the cells in logarithmic growth. (C \& D) Western blot analysis of Cyp40, FKBP52 and FKBP51 protein levels in Karpas 299 (upper) or SUP-M2 (lower) cells treated as in (A) or (B). * indicates a non-specific band in the anti-FKBP51 blot. Quantification of blots are shown to the right and represent the mean and standard deviation of four (C) or three (D) independent experiments. $p$ values comparing untreated cells to cells treated with each concentration of Crizotinib (C) or comparing treated cells at each time point to untreated cells at the $24 \mathrm{~h}$ time point (D) were obtained using paired, one-tailed t-tests. $\S p<0.05,+p<0.01$, \# $p<0.005$.

\section{Cyp40 knock-down does not affect NPM-ALK levels or tyrosine phosphorylation, nor the tyrosine phosphorylation of cellular proteins in ALK+ ALCL}

Cyp40 is primarily noted for its role in co-chaperoning with Hsp90 in complex with steroid hormone receptors [27-30]. However, Cyp40 has also been found in Hsp90/ kinase client complexes. For example, Hsp90/Cyp40 complexes associate with the Lck [36] and Fes [37] tyrosine kinases, and the stability and signalling capacity of ectopically expressed v-Src in S. cerevisiae is dependent on the yeast Cyp40 homolog, Cpr7 [38]. Therefore, we examined whether the decrease in viability due to Cyp40 knockdown could be attributed to a failure of Cyp40 to help Hsp90 stabilize NPM-ALK and/or allow NPM-ALK to signal. We observed no difference in NPM-ALK levels (Figure 6A) or tyrosine phosphorylation (Figure 6B) in Karpas 299 and SUP-M2 cells treated with Cyp40 siRNA compared to control siRNA. Moreover, we saw no significant alteration in the tyrosine phosphorylation of total cellular proteins after Cyp40 knock-down (Figure 6C). However, knock-down of NPM-ALK in these cell lines resulted in a dramatic reduction in the tyrosine phosphorylation of cellular proteins (Figure 6C). We also observed no effect on phosphorylation of STAT3 on tyrosine 705 after knock-down of Cyp40 (Figure 6C). Phosphorylation of STAT3 on this residue is promoted by NPM-ALK signalling [60-62] and is critical for STAT3 DNA binding and transcriptional activity [63-65]. We also found no alteration in the levels of Akt (Figure 6C), which is a known Hsp90 target in this lymphoma [15]. Thus, while Cyp40 is important for the viability of ALK+ ALCL cell lines, our results argue that it does not appear to be influencing viability through regulating NPM-ALK levels or activity, or levels of the Hsp90 client protein Akt.

\section{Discussion}

ALK+ ALCL express the three related immunophilin cochaperones, Cyp40, FKBP51, and FKBP52; however, our findings demonstrate their expression is distinctly regulated in this lymphoma (Figure 7). Signals originating from NPM-ALK promote the expression of Cyp40 and FKBP52, but not FKBP51; whereas the only immunophilin family member regulated by JunB in ALK+ ALCL is
Cyp40. Of note, we were only able to silence JunB expression by $\sim 50 \%$ (see Figure 1 ), so we are likely underestimating the contribution JunB is making to Cyp40 transcription. Since the expression of JunB is promoted by NPM-ALK in ALK+ ALCL $[43,44,46]$, we think it is likely that NPM-ALK promotes the transcription of Cyp40 largely through JunB. However, it is unresolved whether NPM-ALK regulates Cyp40 transcription exclusively through JunB or via a combination of JunBdependent and independent pathways. NPM-ALK knock-down results in a greater reduction in Cyp40 expression that JunB knock-down (compare Figures 1 and 2 to Figure 3), despite a similar reduction in JunB levels in both instances, so we believe it likely that other signalling pathways activated by NPM-ALK also contribute to Cyp40 expression. Moreover, since JunB does not influence FKBP52 expression, this demonstrates NPMALK signalling promotes the transcription of FKBP52 through other downstream effectors. We were surprised by our finding that FKBP51 protein expression was modestly up-regulated in Karpas 299 cells treated with low concentrations of Crizontinib (Figure 4C). However, since we did not observe this increase in FKBP51 protein expression in Crizotinib-treated SUP-M2 cells (Figure 4C), or in Karpas 299 or SUP-M2 cells treated with ALK siRNA (Figure 3C), we are unsure of the significance of this observation.

While this is the first report to show an important role for an immunophilin co-chaperone in lymphoma, several reports have demonstrated that this family of proteins perform critical functions in other malignancies. For example, knock-down of either Cyp40 or FKBP51 in prostate cancer cell lines decreased cellular proliferation; this was particularly evident in androgen-dependent cell lines where these co-chaperones promote the transcriptional activity of the androgen receptor [39]. Metastatic melanoma has high levels of FKBP51, and knock-down of FKBP51 sensitized the SAN melanoma cell line to ionizing radiation [66]. This response was postulated to be due to decreased anti-apoptotic signalling through NF-kB in response to reduced FKBP51 levels [66]. In contrast, reducing the expression of FKBP51 in breast, lung, and pancreatic cancer cell lines resulted in reduced sensitivity 

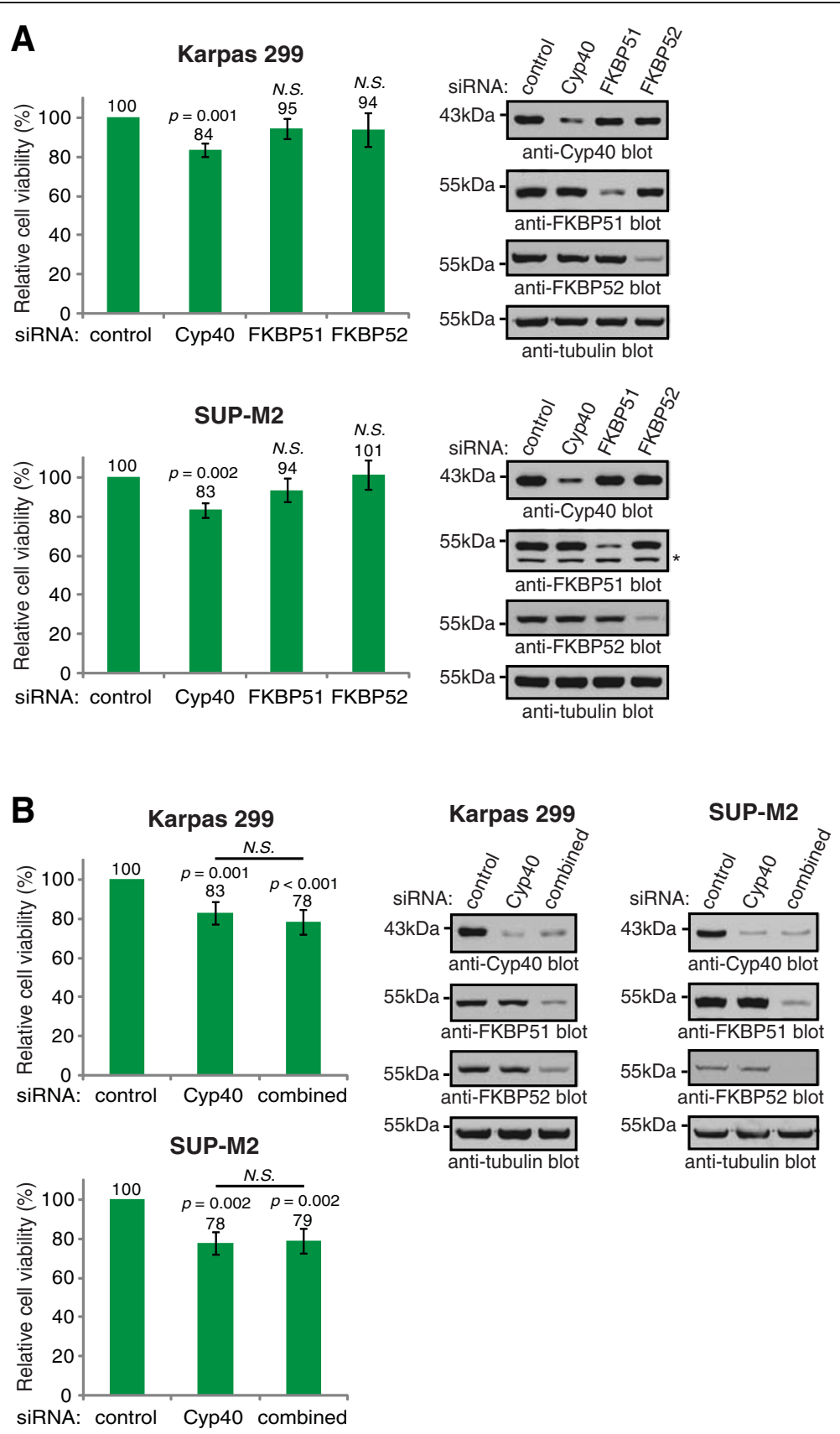

Figure 5 Cyp40, but not FKBP51 or FKBP52, promotes ALK+ ALCL viability. (A) The viability of Karpas 299 (upper) or SUP-M2 (lower) cells transfected with the indicated pooled siRNAs was measured using the MTS assay (left). Western blots (right) demonstrate the silencing efficiency of the targeted proteins. * indicates a non-specific band in the anti-FKBP51 blot. (B) Viability of Karpas 299 (upper left) or SUP-M2 (lower left) cells transfected with the indicated pooled siRNAs. Combined siRNA consists of siRNAs to target the three immunophilin co-chaperones, Cyp40, FKBP51 and FKBP52. Western blots (right) demonstrate the level of silencing of the indicated proteins. Quantification represents the mean and standard deviation of four independent experiments. $p$ values comparing cells transfected with the indicated siRNA to those transfected with control siRNA were obtained using paired, one-tailed $t$-tests. $p$ values comparing cells transfected with Cyp40 or combined siRNA were obtained using unpaired, one-tailed t-tests. N.S. indicates a $p$ value $>0.05$.

to chemotherapeutic agents [67]. It was suggested in this study that activation of Akt was partially responsible for this decreased sensitivity. Thus, the immunophilin cochaperones perform important functions in a number of cancers, and may represent attractive therapeutic targets in some malignancies.

An important unanswered question arising from our study is why reducing Cyp40 expression in ALK+ ALCL 
A
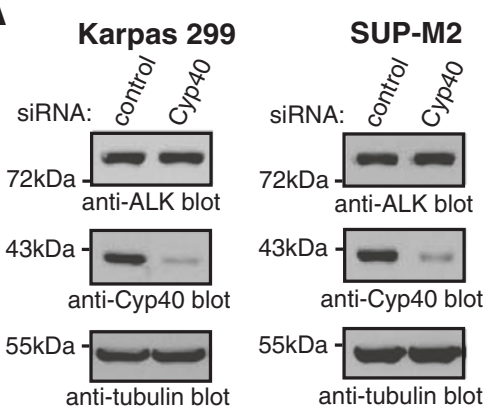

B

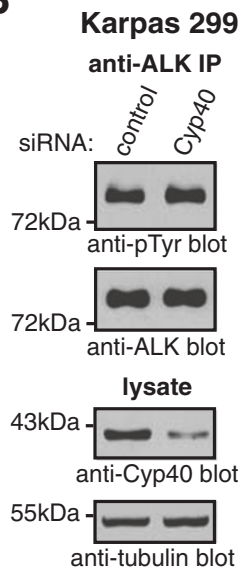

SUP-M2
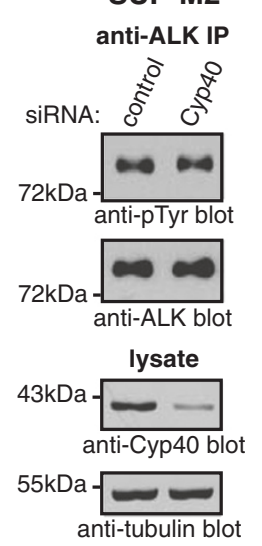

c

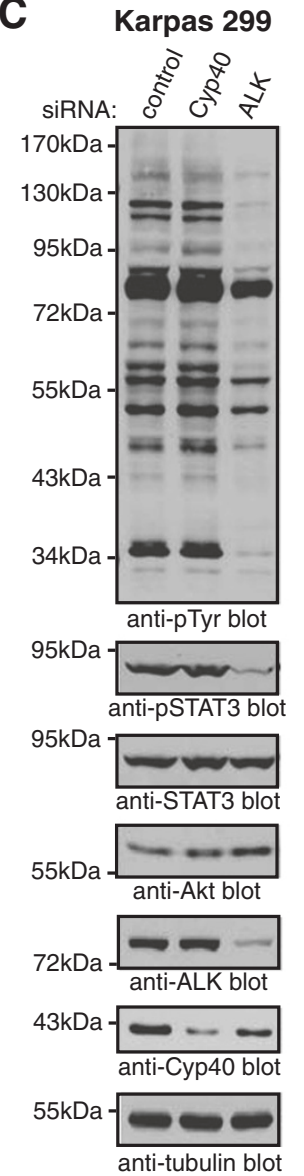

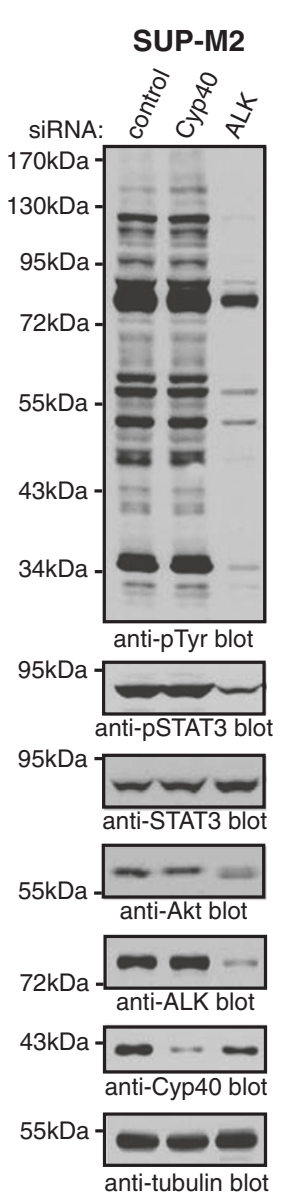

Figure 6 Cyp40 does not regulate NPM-ALK expression or appear to influence NPM-ALK signalling. (A) Western blot analysis of NPM-ALK protein levels (anti-ALK blots) in lysates collected from Karpas 299 and SUP-M2 cells after transfection with pooled control or Cyp40 siRNA. (B) The tyrosine phosphorylation status of NPM-ALK was analyzed by immunoprecipitation of NPM-ALK (anti-ALK IP) followed by antiphosphotyrosine (pTyr) western blotting in cells treated as in (A). (C) The tyrosine phosphorylation of total cellular proteins (anti-pTyr blot), the phosphorylation status of STAT3 (anti-pSTAT3 blot), and total Akt levels were analyzed by western blotting in lysates from cells transfected with pooled control, Cyp40, or ALK siRNA.

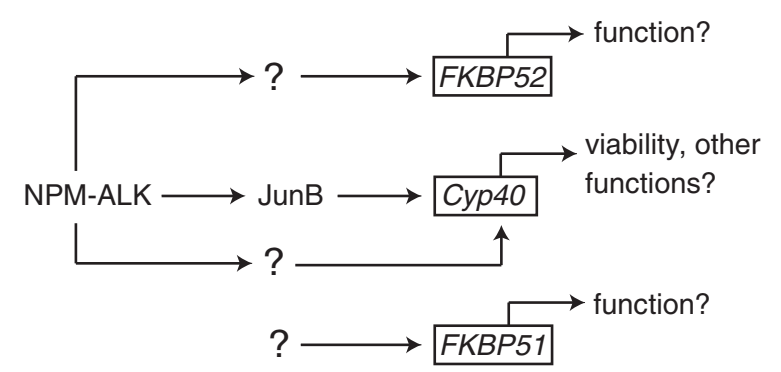

Figure 7 Pathways influencing expression of the immunophilin family of co-chaperones in ALK+ ALCL. The transcription of Cyp40 is promoted by signals initiated by NPM-ALK, and we postulate that up-regulation of JunB by NPM-ALK accounts for much of the increase in Cyp40 transcription. However, additional signalling pathways activated by this oncoprotein likely also contribute to Cyp40 expression. The transcription of FKBP52 is promoted by NPM$A L K$ in $A L K+A L C L$, but in a manner that is independent of JunB. Although expressed in ALK+ ALCL, FKBP51 expression is not regulated by NPM-ALK or JunB in this lymphoma. cell lines resulted in reduced viability (Figure 5). Specific experiments to determine whether this is an increase in apoptosis, a decrease in proliferation, or combination of both of these processes have been inconclusive. This decrease in viability does not appear to be due to an impairment of NPM-ALK activity (Figure 6), and suggests that the dysregulation of another protein(s) is important for this phenotype. In addition to steroid hormone receptors and kinases, Cyp40 is known to associate with a number of other proteins with a variety of cellular functions including the c-Myb transcription factor [68], mutant forms of p53 [69], and the RACK1 scaffolding protein [70]. Also, a genetic study in Arabidopsis identified an important role for the Cyp40 orthologue, SQUINT, in microRNA biogenesis [71]. Thus, there are several cellular activities whose disruption could account for the decreased viability observed when Cyp40 is knocked down in ALK+ ALCL cell lines. Regardless of 
the exact cellular activity or activities regulated by Cyp40 that is important for the viability of ALK+ ALCL cell lines, our results clearly show these activities are not redundant with FKBP51 and FKBP52.

Our results show that Cyp40 does not regulate NPMALK levels or activity (Figure 6), but it is possible that other co-chaperones could be working with Hsp90 to regulate NPM-ALK activity. There are currently more than 20 known Hsp90 co-chaperones [19,20]. One of these proteins, $\mathrm{Cdc} 37$, co-chaperones for many kinase client proteins including Erb-B2, c-Raf, CDK4, CDK6 and Akt [72]. Cdc37 was identified by mass spectrometry as an NPM-ALK associated protein [73], and has also been shown to complex with EML4-ALK in NSCLC [22]. These studies however, did not examine whether these interactions are important for the activity of the respective ALK fusion proteins. We are currently investigating whether $\mathrm{Cdc} 37$ or other Hsp90 co-chaperones influence NPM-ALK activity. If a co-chaperone protein that cooperates with Hsp90 to regulate NPM-ALK can be identified, it could represent a potential drug target to treat ALK+ ALCL, and other cancers expressing ALK fusion proteins, especially in situations where ALK mutations have resulted in resistance to conventional ALK inhibitors.

\section{Conclusions}

The Hsp90 chaperone protein regulates the NPM-ALK oncoprotein and other signalling molecules that promote proliferation and survival in ALK+ ALCL. Co-chaperone proteins are important co-factors of Hsp90, and in this study we examined the regulation and function of the immunophilin co-chaperones in ALK+ ALCL. We show that NPM-ALK is required for the expression of the immunophilin co-chaperones, Cyp40 and FKPB52, but not FKBP51 in ALK+ ALCL. Our findings further demonstrate that regulation of Cyp40 and FKPB52 by NPMALK is distinct, given that Cyp40 expression in ALK+ ALCL is promoted by the JunB transcription factor, whereas FKBP52 expression is not. Importantly, this is the first study demonstrating that signalling by an oncogenic tyrosine kinase promotes the expression of an immunophilin family co-chaperone, and identifies Cyp40 as a novel JunB transcriptional target. Finally, we demonstrate that Cyp40 promotes the viability of ALK+ ALCL cell lines in a manner that is independent of the other related immunophilin co-chaperones.

\section{Competing interests}

The authors declare that they have no competing interests.

\section{Acknowledgements}

The authors would like to thank Jason Lee and Dr. Troy Baldwin for helpful discussions relating to this work, and Jason Lee and Brandon Maser for critically reading the manuscript.
This work was supported by operating grants from the British Columbia Proteomics Network, the Natural Sciences and Engineering Research Council of Canada (NSERC), the Alberta Cancer Foundation/Alberta Innovates Health Solutions, and start-up funds from the University of Alberta (all to RJI). Part of this work was also funded by a grant from the University Hospital Foundation (to JTCB). JDP is a recipient of studentships from NSERC and the Alberta Cancer Foundation.

\section{Author details}

'Department of Medical Microbiology and Immunology, University of Alberta, Edmonton T6G 2E1, Canada. ${ }^{2}$ Department of Laboratory Medicine and Pathology, University of Alberta, Edmonton T6G 2B7, Canada.

\section{Authors' contributions}

J.D.P, R.J.I, J.T.C.B, and R.L were involved in the conception and design of experiments. J.D.P performed the majority of experiments, and Z.M. performed some of the luciferase assays. J.D.P. and R.J.I drafted the original manuscript. All authors read and approved the final manuscript.

Received: 26 March 2012 Accepted: 8 June 2012

Published: 8 June 2012

\section{References}

1. Delsol G, Falini B, Muller-Hermelink H, Campo E, Jaffe E, Gascoyne R, Stein H, Kinney M: Anaplastic Large Cell Lymphoma (ALCL), ALK-positive. In International Agency for Research on Cancer (IARC). Edited by Swerdlow S, Campo E, Harris N, Jaffe E, Pileri S, Stein H, Thiele J, Vardiman J. Lyon, Francec: IARC Press; 2008:312-316.

2. Amin HM, Lai R: Pathobiology of ALK+ anaplastic large-cell lymphoma. Blood 2007, 110:2259-2267.

3. Chiarle R, Voena C, Ambrogio C, Piva R, Inghirami G: The anaplastic lymphoma kinase in the pathogenesis of cancer. Nat Rev Cancer 2008, 8:11-23.

4. Soda M, Takada S, Takeuchi K, Choi YL, Enomoto M, Ueno T, Haruta H, Hamada T, Yamashita Y, Ishikawa Y, et al: A mouse model for EML4-ALKpositive lung cancer. Proc Natl Acad Sci U S A 2008, 105:19893-19897.

5. Choi YL, Takeuchi K, Soda M, Inamura K, Togashi Y, Hatano S, Enomoto M, Hamada T, Haruta $H$, Watanabe $H$, et al: Identification of novel isoforms of the EML4-ALK transforming gene in non-small cell lung cancer. Cancer Res 2008, 68:4971-4976.

6. Koivunen JP, Mermel C, Zejnullahu K, Murphy C, Lifshits E, Holmes AJ, Choi HG, Kim J, Chiang D, Thomas R, et al: EML4-ALK fusion gene and efficacy of an ALK kinase inhibitor in lung cancer. Clin Cancer Res 2008, 14:42754283.

7. Kwak EL, Bang YJ, Camidge DR, Shaw AT, Solomon B, Maki RG, Ou SH, Dezube BJ, Janne PA, Costa DB, et al: Anaplastic lymphoma kinase inhibition in non-small-cell lung cancer. N Engl J Med 2010, 363:16931703.

8. Lawrence B, Perez-Atayde A, Hibbard MK, Rubin BP, Dal Cin P, Pinkus JL, Pinkus GS, Xiao S, Yi ES, Fletcher CD, Fletcher JA: TPM3-ALK and TPM4-ALK oncogenes in inflammatory myofibroblastic tumors. Am J Pathol 2000, 157:377-384.

9. Griffin CA, Hawkins AL, Dvorak C, Henkle C, Ellingham T, Perlman EJ: Recurrent involvement of 2p23 in inflammatory myofibroblastic tumors. Cancer Res 1999, 59:2776-2780.

10. Butrynski JE, D’Adamo DR, Hornick JL, Dal Cin P, Antonescu CR, Jhanwar SC, Ladanyi M, Capelletti M, Rodig SJ, Ramaiya N, et al: Crizotinib in ALKrearranged inflammatory myofibroblastic tumor. N Engl J Med 2010, 363:1727-1733.

11. Morris SW, Kirstein MN, Valentine MB, Dittmer KG, Shapiro DN, Saltman DL, Look AT: Fusion of a kinase gene, ALK, to a nucleolar protein gene, NPM, in non-Hodgkin's lymphoma. Science 1994, 263:1281-1284.

12. Shiota M, Fujimoto J, Semba T, Satoh H, Yamamoto T, Mori S: Hyperphosphorylation of a novel $80 \mathrm{kDa}$ protein-tyrosine kinase similar to Ltk in a human Ki-1 lymphoma cell line, AMS3. Oncogene 1994, 9:1567-1574.

13. Fujimoto J, Shiota M, Iwahara T, Seki N, Satoh H, Mori S, Yamamoto T: Characterization of the transforming activity of p80, a hyperphosphorylated protein in a Ki-1 lymphoma cell line with 
chromosomal translocation t(2;5). Proc Natl Acad Sci U S A 1996, 93:41814186.

14. Bischof D, Pulford K, Mason DY, Morris SW: Role of the nucleophosmin (NPM) portion of the non-Hodgkin's lymphoma-associated NPManaplastic lymphoma kinase fusion protein in oncogenesis. Mol Cell Biol 1997, 17:2312-2325.

15. Georgakis GV, Li Y, Rassidakis GZ, Medeiros $\sqcup$, Younes A: The HSP90 inhibitor 17-AAG synergizes with doxorubicin and U0126 in anaplastic large cell lymphoma irrespective of ALK expression. Exp Hematol 2006, 34:1670-1679.

16. Bonvini P, Gastaldi T, Falini B, Rosolen A: Nucleophosmin-anaplastic lymphoma kinase (NPM-ALK), a novel Hsp90-client tyrosine kinase: down-regulation of NPM-ALK expression and tyrosine phosphorylation in $\mathrm{ALK}(+) \mathrm{CD} 30(+)$ lymphoma cells by the Hsp90 antagonist 17allylamino,17-demethoxygeldanamycin. Cancer Res 2002, 62:1559-1566.

17. Bonvini P, Dalla Rosa H, Vignes N, Rosolen A: Ubiquitination and proteasomal degradation of nucleophosmin-anaplastic lymphoma kinase induced by 17-allylamino-demethoxygeldanamycin: role of the cochaperone carboxyl heat shock protein 70-interacting protein. Cancer Res 2004, 64:3256-3264.

18. Schumacher JA, Crockett DK, Elenitoba-Johnson KS, Lim MS: Proteome-wide changes induced by the Hsp90 inhibitor, geldanamycin in anaplastic large cell lymphoma cells. Proteomics 2007, 7:2603-2616.

19. Trepel J, Mollapour M, Giaccone G, Neckers L: Targeting the dynamic HSP90 complex in cancer. Nat Rev Cancer 2010, 10:537-549.

20. Taipale M, Jarosz DF, Lindquist S: HSP90 at the hub of protein homeostasis: emerging mechanistic insights. Nat Rev Mol Cell Biol 2010, 11:515-528.

21. Theodoraki MA, Kunjappu M, Sternberg DW, Caplan AJ: Akt shows variable sensitivity to an Hsp90 inhibitor depending on cell context. Exp Cell Res 2007, 313:3851-3858.

22. Chen Z, Sasaki T, Tan X, Carretero J, Shimamura T, Li D, Xu C, Wang Y, Adelmant GO, Capelletti M, et al: Inhibition of ALK, PI3K/MEK, and HSP90 in murine lung adenocarcinoma induced by EML4-ALK fusion oncogene. Cancer Res 2010, 70:9827-9836.

23. Normant E, Paez G, West KA, Lim AR, Slocum KL, Tunkey C, McDougall J, Wylie AA, Robison K, Caliri K, et al: The Hsp90 inhibitor IPI-504 rapidly lowers EML4ALK levels and induces tumor regression in ALK-driven NSCLC models. Oncogene 2011, 30:2581-2586.

24. Katayama R, Khan TM, Benes C, Lifshits E, Ebi H, Rivera VM, Shakespeare WC, lafrate AJ, Engelman JA, Shaw AT: Therapeutic strategies to overcome crizotinib resistance in non-small cell lung cancers harboring the fusion oncogene EML4-ALK. Proc Natl Acad Sci U S A 2011, 108:7535-7540.

25. Sequist LV, Gettinger S, Senzer NN, Martins RG, Janne PA, Lilenbaum R, Gray JE, lafrate AJ, Katayama R, Hafeez N, et al: Activity of IPI-504, a novel heatshock protein 90 inhibitor, in patients with molecularly defined nonsmall-cell lung cancer. J Clin Oncol 2010, 28:4953-4960.

26. Sasaki T, Okuda K, Zheng W, Butrynski J, Capelletti M, Wang L, Gray NS, Wilner K, Christensen JG, Demetri G, et al: The neuroblastoma-associated F1174L ALK mutation causes resistance to an ALK kinase inhibitor in ALK-translocated cancers. Cancer Res 2010, 70:10038-10043.

27. Ratajczak T, Ward BK, Minchin RF: Immunophilin chaperones in steroid receptor signalling. Curr Top Med Chem 2003, 3:1348-1357.

28. Ratajczak T, Ward BK, Cluning C, Allan RK: Cyclophilin 40: an Hsp90cochaperone associated with apo-steroid receptors. Int J Biochem Cell Biol 2009, 41:1652-1655.

29. Davies TH, Sanchez ER: Fkbp52. Int J Biochem Cell Biol 2005, 37:42-47.

30. Li L, Lou Z, Wang L: The role of FKBP5 in cancer aetiology and chemoresistance. Br J Cancer 2011, 104:19-23.

31. Denny WB, Valentine DL, Reynolds PD, Smith DF, Scammell JG: Squirrel monkey immunophilin FKBP51 is a potent inhibitor of glucocorticoid receptor binding. Endocrinology 2000, 141:4107-4113.

32. Riggs DL, Roberts PJ, Chirillo SC, Cheung-Flynn J, Prapapanich V, Ratajczak T, Gaber R, Picard D, Smith DF: The Hsp90-binding peptidylprolyl isomerase FKBP52 potentiates glucocorticoid signaling in vivo. EMBO J 2003, 22:1158-1167.

33. Wochnik GM, Ruegg J, Abel GA, Schmidt U, Holsboer F, Rein T: FK506binding proteins 51 and 52 differentially regulate dynein interaction and nuclear translocation of the glucocorticoid receptor in mammalian cells. J Biol Chem 2005, 280:4609-4616.

34. Warrier M, Hinds TD Jr, Ledford KJ, Cash HA, Patel PR, Bowman TA, Stechschulte LA, Yong W, Shou W, Najjar SM, Sanchez ER: Susceptibility to diet-induced hepatic steatosis and glucocorticoid resistance in FK506-binding protein 52deficient mice. Endocrinology 2010, 151:3225-3236.

35. Davies TH, Ning YM, Sanchez ER: Differential control of glucocorticoid receptor hormone-binding function by tetratricopeptide repeat (TPR) proteins and the immunosuppressive ligand FK506. Biochemistry 2005, 44:2030-2038.

36. Scroggins BT, Prince T, Shao J, Uma S, Huang W, Guo Y, Yun BG, Hedman K, Matts RL, Hartson SD: High affinity binding of Hsp90 is triggered by multiple discrete segments of its kinase clients. Biochemistry 2003, 42:12550-12561.

37. Nair SC, Toran EJ, Rimerman RA, Hjermstad S, Smithgall TE, Smith DF: A pathway of multi-chaperone interactions common to diverse regulatory proteins: estrogen receptor, Fes tyrosine kinase, heat shock transcription factor Hsf1, and the aryl hydrocarbon receptor. Cell Stress Chaperones 1996, 1:237-250.

38. Duina AA, Chang HC, Marsh JA, Lindquist S, Gaber RF: A cyclophilin function in Hsp90-dependent signal transduction. Science 1996, 274:1713-1715.

39. Periyasamy S, Hinds T Jr, Shemshedini L, Shou W, Sanchez ER: FKBP51 and Cyp40 are positive regulators of androgen-dependent prostate cancer cell growth and the targets of FK506 and cyclosporin A. Oncogene 2010, 29:1691-1701.

40. Mathas S, Hinz M, Anagnostopoulos I, Krappmann D, Lietz A, Jundt F, Bommert K, Mechta-Grigoriou F, Stein H, Dorken B, Scheidereit C: Aberrantly expressed c-Jun and JunB are a hallmark of Hodgkin lymphoma cells, stimulate proliferation and synergize with NF-kappa B. EMBO J 2002, 21:4104-4113.

41. Rassidakis GZ, Thomaides A, Atwell C, Ford R, Jones D, Claret FX, Medeiros $L J$ : JunB expression is a common feature of CD30+ lymphomas and lymphomatoid papulosis. Mod Pathol 2005, 18:1365-1370.

42. Szremska AP, Kenner L, Weisz E, Ott RG, Passegue E, Artwohl M, Freissmuth $M$, Stoxreiter R, Theussl HC, Parzer SB, et al: JunB inhibits proliferation and transformation in B-lymphoid cells. Blood 2003, 102:4159-4165.

43. Staber PB, Vesely P, Haq N, Ott RG, Funato K, Bambach I, Fuchs C, Schauer S, Linkesch W, Hrzenjak A, et al: The oncoprotein NPM-ALK of anaplastic large-cell lymphoma induces JUNB transcription via ERK1/2 and JunB translation via mTOR signaling. Blood 2007, 110:3374-3383.

44. Hsu FY, Johnston PB, Burke KA, Zhao Y: The expression of CD30 in anaplastic large cell lymphoma is regulated by nucleophosminanaplastic lymphoma kinase-mediated JunB level in a cell type-specific manner. Cancer Res 2006, 66:9002-9008.

45. Watanabe M, Sasaki M, Itoh K, Higashihara M, Umezawa K, Kadin ME, Abraham $L$, Watanabe T, Horie R: JunB induced by constitutive CD30extracellular signal-regulated kinase $1 / 2$ mitogen-activated protein kinase signaling activates the CD30 promoter in anaplastic large cell lymphoma and reed-sternberg cells of Hodgkin lymphoma. Cancer Res 2005, 65:7628-7634.

46. Pearson JD, Lee JK, Bacani JT, Lai R, Ingham RJ: NPM-ALK and the JunB transcription factor regulate the expression of cytotoxic molecules in ALK-positive, anaplastic large cell lymphoma. Int J Clin Exp Pathol 2011, 4:124-133.

47. Kinney MC, Higgins RA, Medina EA: Anaplastic large cell lymphoma: twenty-five years of discovery. Arch Pathol Lab Med 2011, 135:19-43.

48. Christensen JG, Zou HY, Arango ME, Li Q, Lee JH, McDonnell SR, Yamazaki S, Alton GR, Mroczkowski B, Los G: Cytoreductive antitumor activity of PF2341066, a novel inhibitor of anaplastic lymphoma kinase and c-Met, in experimental models of anaplastic large-cell lymphoma. Mol Cancer Ther 2007, 6:3314-3322

49. McDermott U, lafrate AJ, Gray NS, Shioda T, Classon M, Maheswaran S, Zhou W, Choi HG, Smith SL, Dowell L, et al: Genomic alterations of anaplastic lymphoma kinase may sensitize tumors to anaplastic lymphoma kinase inhibitors. Cancer Res 2008, 68:3389-3395.

50. Ingham RJ, Raaijmakers J, Lim CS, Mbamalu G, Gish G, Chen F, Matskova L, Ernberg I, Winberg G, Pawson T: The Epstein-Barr virus protein, latent membrane protein $2 \mathrm{~A}$, co-opts tyrosine kinases used by the $\mathrm{T}$ cell receptor. J Biol Chem 2005, 280:34133-34142.

51. Livak KJ, Schmittgen TD: Analysis of relative gene expression data using real-time quantitative PCR and the 2(-Delta Delta C(T)) Method. Methods 2001, 25:402-408.

52. Kumar P, Ward BK, Minchin RF, Ratajczak T: Regulation of the Hsp90binding immunophilin, cyclophilin 40 , is mediated by multiple sites for GA-binding protein (GABP). Cell Stress Chaperones 2001, 6:78-91. 
53. Gambacorti-Passerini C, Messa C, Pogliani EM: Crizotinib in anaplastic large-cell lymphoma. N Engl J Med 2011, 364:775-776.

54. Kimura H, Nakajima T, Takeuchi K, Soda M, Mano H, lizasa T, Matsui Y, Yoshino M, Shingyoji M, Itakura M, et al: ALK fusion gene positive lung cancer and 3 cases treated with an inhibitor for ALK kinase activity. Lung Cancer 2012, 75:66-72.

55. Kijima T, Takeuchi K, Tetsumoto S, Shimada K, Takahashi R, Hirata H, Nagatomo I, Hoshino S, Takeda Y, Kida H, et al: Favorable response to crizotinib in three patients with echinoderm microtubule-associated protein-like 4-anaplastic lymphoma kinase fusion-type oncogenepositive non-small cell lung cancer. Cancer Sci 2011, 102:1602-1604.

56. Tartari CJ, Gunby RH, Coluccia AM, Sottocornola R, Cimbro B, Scapozza L, Donella-Deana A, Pinna LA, Gambacorti-Passerini C: Characterization of some molecular mechanisms governing autoactivation of the catalytic domain of the anaplastic lymphoma kinase. J Biol Chem 2008, 283:37433750 .

57. Wang $P$, Wu F, Ma Y, Li L, Lai R, Young LC: Functional characterization of the kinase activation loop in nucleophosmin (NPM)-anaplastic lymphoma kinase (ALK) using tandem affinity purification and liquid chromatography-mass spectrometry. J Biol Chem 2010, 285:95-103.

58. Nair SC, Rimerman RA, Toran EJ, Chen S, Prapapanich V, Butts RN, Smith DF: Molecular cloning of human FKBP51 and comparisons of immunophilin interactions with Hsp90 and progesterone receptor. Mol Cell Biol 1997 17:594-603.

59. Barent RL, Nair SC, Carr DC, Ruan Y, Rimerman RA, Fulton J, Zhang Y, Smith DF: Analysis of FKBP51/FKBP52 chimeras and mutants for Hsp90 binding and association with progesterone receptor complexes. Mol Endocrinol 1998, 12:342-354.

60. Zhang Q, Raghunath PN, Xue L, Majewski M, Carpentieri DF, Odum N, Morris S, Skorski T, Wasik MA: Multilevel dysregulation of STAT3 activation in anaplastic lymphoma kinase-positive T/null-cell lymphoma. J Immunol 2002, 168:466-474.

61. Zamo A, Chiarle R, Piva R, Howes J, Fan Y, Chilosi M, Levy DE, Inghirami G: Anaplastic lymphoma kinase (ALK) activates Stat3 and protects hematopoietic cells from cell death. Oncogene 2002, 21:1038-1047.

62. Wan W, Albom MS, Lu L, Quail MR, Becknell NC, Weinberg LR, Reddy DR, Holskin BP, Angeles TS, Underiner TL, et al: Anaplastic lymphoma kinase activity is essential for the proliferation and survival of anaplastic largecell lymphoma cells. Blood 2006, 107:1617-1623.

63. Schaefer TS, Sanders LK, Park OK, Nathans D: Functional differences between Stat3alpha and Stat3beta. Mol Cell Biol 1997, 17:5307-5316.

64. Schaefer LK, Wang S, Schaefer TS: c-Src activates the DNA binding and transcriptional activity of Stat3 molecules: serine 727 is not required for transcriptional activation under certain circumstances. Biochem Biophys Res Commun 1999, 266:481-487.

65. Kaptein A, Paillard V, Saunders M: Dominant negative stat3 mutant inhibits interleukin-6-induced Jak-STAT signal transduction. J Bio/ Chem 1996, 271:5961-5964.

66. Romano S, D'Angelillo A, Pacelli R, Staibano S, De Luna E, Bisogni R, Eskelinen EL, Mascolo M, Cali G, Arra C, Romano MF: Role of FK506-binding protein 51 in the control of apoptosis of irradiated melanoma cells. Cell Death Differ 2010, 17:145-157.

67. Pei H, Li L, Fridley BL, Jenkins GD, Kalari KR, Lingle W, Petersen G, Lou Z, Wang L: FKBP51 affects cancer cell response to chemotherapy by negatively regulating Akt. Cancer Cell 2009, 16:259-266.

68. Leverson JD, Ness SA: Point mutations in v-Myb disrupt a cyclophilincatalyzed negative regulatory mechanism. Mol Cell 1998, 1:203-211.

69. Whitesell L, Sutphin PD, Pulcini EJ, Martinez JD, Cook PH: The physical association of multiple molecular chaperone proteins with mutant p53 is altered by geldanamycin, an hsp90-binding agent. Mol Cell Biol 1998, 18:1517-1524.

70. Park MS, Chu F, Xie J, Wang Y, Bhattacharya P, Chan WK: Identification of cyclophilin-40-interacting proteins reveals potential cellular function of cyclophilin-40. Anal Biochem 2011, 410:257-265.

71. Smith MR, Willmann MR, Wu G, Berardini TZ, Moller B, Weijers D, Poethig RS: Cyclophilin 40 is required for microRNA activity in Arabidopsis. Proc Natl Acad Sci U S A 2009, 106:5424-5429.

72. Smith JR, Clarke PA, de Billy E, Workman P: Silencing the cochaperone CDC37 destabilizes kinase clients and sensitizes cancer cells to HSP90 inhibitors. Oncogene 2009, 28:157-169.
73. Wu F, Wang P, Young LC, Lai R, Li L: Proteome-wide identification of novel binding partners to the oncogenic fusion gene protein, NPM-ALK, using tandem affinity purification and mass spectrometry. Am J Pathol 2009, 174:361-370.

doi:10.1186/1471-2407-12-229

Cite this article as: Pearson et al:: The heat shock protein-90 cochaperone, Cyclophilin 40, promotes ALK-positive, anaplastic large cell lymphoma viability and its expression is regulated by the NPM-ALK oncoprotein. BMC Cancer 2012 12:229.

\section{Submit your next manuscript to BioMed Central and take full advantage of:}

- Convenient online submission

- Thorough peer review

- No space constraints or color figure charges

- Immediate publication on acceptance

- Inclusion in PubMed, CAS, Scopus and Google Scholar

- Research which is freely available for redistribution 